\title{
A modified version of the Greek Simplified Medication Adherence Questionnaire for hemodialysis patients
}

\author{
Victoria Alikari, ${ }^{1}$ Vasiliki Matziou, ${ }^{2}$ Maria Tsironi, ${ }^{1}$ Natasa Kollia, ${ }^{3}$ Paraskevi Theofilou, ${ }^{1}$ \\ Adamantia Aroni, ${ }^{1}$ Evangelos Fradelos, ${ }^{1}$ Sofia Zyga ${ }^{1}$ \\ ${ }^{1}$ Department of Nursing, University of Peloponnese; ${ }^{2}$ Department of Nursing, National and \\ Kapodistrian University of Athens; ${ }^{3}$ Department of Nutrition and Dietetics, School of Health \\ Science and Education, Harokopio University, Athens, Greece
}

\begin{abstract}
Non-adherence to the therapeutic regimen is an increasingly growing problem especially among patients undergoing hemodialysis. The aim of this study was to modify the Greek version of Simplified Medication Adherence Questionnaire (GR-SMAQ) for patients undergoing hemodialysis (GR-SMAQ-HD) and explore its validity and reliability. Between June 2016 and November 2016 a group of patients undergoing hemodialysis $(\mathrm{N}=107)$ completed the Greek version of SMAQ. The study was carried out in three Dialysis Units of Hospitals of Athens and Peloponnese region, Greece. The form of GR-SMAQ was modified specifically for renal patients while four additional items were added so as the tool study all aspects of adherence to hemodialysis regimen.
\end{abstract}

\footnotetext{
Correspondence: Victoria Alikari, Nursing Department, Faculty of Human Movement and Quality of Life, University of Peloponnese, Sparta, Laconia, Greece.

Tel.: +30.693.6168825.

E-mail: vicalikari@gmail.com

Key words: adherence; SMAQ; hemodialysis; validity.

Acknowledgements: the authors would like to thank patients on hemodialysis, nursing staff and the Scientific Councils of the Dialysis Unit "Medifil", Athens, "Molaoi" Hospital, and Hospital of Tripolis "Evagelistria", Peloponnese, Greece.

Contributions: AV, study conception, design and coordination, interviews undertaking, literature review, paper writing; VM, project leading, design and coordination; MT, design and coordination; NK, data analysis, manuscript drafting; PT, interviews undertaking, design and coordination; AA, interviews undertaking, literature review; EF, interviews undertaking, manuscript drafting, literature review; SZ, project leading, manuscript drafting.
}

Conflict of interest: the authors declare no potential conflict of interest.

Received for publication: 14 February 2017.

Revision received: 18 April 2017.

Accepted for publication: 27 April 2017.

This work is licensed under a Creative Commons AttributionNonCommercial 4.0 International License (CC BY-NC 4.0).

CCopyright V. Alikari et al., 2017

Licensee PAGEPress, Italy

Health Psychology Research 2017; 5:6647

doi:10.4081/hpr.2017.6647
Construct validity was checked through exploratory factor analysis with principal Component Analysis with the Equamax method. Test-retest reliability and internal consistency were tested. Statistical analysis was performed using the IBM SPSS Statistics version 21 . The significance level was set up at $5 \%$. The Greek version of SMAQ for patients undergoing hemodialysis includes eight questions. Three factors emerged from factor analysis. Cronbach's $\alpha$ coefficient was 0.742 for the whole scale and for each subscale was for Medication Adherence 0.75, for Attendance at hemodialysis session 0.856 and for Diet/Fluid restriction was 0.717 . The total mean score was $6.29( \pm 1.82)$. GR-SMAQ-HD is a reliable and valuable tool that can be used by hemodialysis nurses and students of nursing for detection of adherence levels in clinical practice.

\section{Introduction}

End Stage Renal Disease (ESRD) undergoing hemodialysis (HD) is one of the chronic diseases with effects on patient's quality of life. Patients undergoing hemodialysis must adhere to a very restricted diet and fluid intake. These patients require four hours of dialysis sessions three times a week at dialysis unit. These results in a loss of time that affects employment, leisure and social relationships (Ikonomou et al., 2015). Patients undergoing HD experience multiple problems such as sodium and water retention (Lee, 2012), hyperphosphatemia (Karamanidou, Clatworthy, Weinman, \& Horne, 2008), hyperkalemia (Bellizzi et al., 2016), hypertension, anemia (Erlingmark, Hedström, \& Lindberg, 2016), fatigue (Zyga et al., 2015) heart disease (Segall, Nistor, \& Covic, 2014) or diabetes mellitus (van der Meer, Ruggenenti, \& Remuzzi, 2010). The management of such health issues involves several variations in the patient's lifestyle. The success of treatment depends largely on patient adherence to stringent recommended regimen. Non-adherence to therapeutic recommendations can have untoward effects on quality of life (Ibrahim, Hossam, \& Belal 2015), increased morbidity, healthcare costs and mortality. Overall, it is estimated that approximately $50 \%$ of patients on dialysis are poorly compliant with their treatment (Kammerer, Garry, Hartigan, Carter, \& Erlich, 2007).

\section{Background}

According to the National Kidney Foundation and Kidney Disease Outcomes Quality Initiative (Estrella et al., 2013) nonadherence among patients undergoing HD include: failure or shorten the session b) excessive intake of fluids and foods containing potassium and phosphorus and c) non-adherence to medication treatment. Non-adherence to attendance at HD session, pre- 
scribed medications, fluid restrictions, and dietary intake range from $0 \%$ to $32.3 \%, 1.2 \%$ to $81 \%, 3.4 \%$ to $74 \%$, and $1.2 \%$ to $82.4 \%$, respectively (Durose, Holdsworth, Watson, \& Przygrodzka, 2004; Kutner, Zhang, \& McClellan, 2002).

\section{The effect of non-adherence in hemodialysis regimen}

Adherence to prescribed hemodialysis regimen is a critical factor for obtaining good therapeutic results for patients undergoing HD and contributes to reducing morbidity, mortality and hemodialysis side effects (muscle cramps, malnutrition, sepsis, infections) (Lòpez-Gòmez, Villaverde, Jofre, \& Pérez-García, 2005). Nonadherence to fluid restriction can cause anxiety, panic, dyspnea, hypertension and pulmonary edema. Repeated excessive fluid intake periods burden on the cardiovascular system. Although hypertension is a risk factor for cardiovascular disease, the results of studies on the relationship between the nonadherence to fluid restriction and mortality were positive (Szczech et al., 2003) or negative (Lòpez-Gòmez, et al., 2005). These results may indicate that other variables, such as nutritional status and age may act as mediators (Szczech et al., 2003; Lòpez-Gòmez et al., 2005).

Non-adherence to diet and medication regimen can lead to chronically elevated serum phosphorus levels, which play a main role in the development of secondary hyperparathyroidism and renal osteodystrophy. Elevated phosphate levels may, also, increase the risk for coronary heart disease even in young patients (Goodman, 2002) leading to a significantly increased risk of mortality (Block et al., 2004). Severe hyperkalemia is common among patients undergoing HD with an inclination to suicide or who ignore proper dietary regimen.

The skipping or shortening of the hemodialysis session reduces the adequacy of dialysis. The skipping of at least one dialysis session per month has been associated with $25-30 \%$ higher risk of death while the abbreviation for more than 10 minutes $(\geq 3$ times per month) has, also, been associated with increased mortality (Leggat et al., 1998).

\section{Measures of patient adherence}

Patient adherence is measured in various ways in clinical practice. The measurement methods used may be objective or subjective. Pills counting are usually carried out in patient's home or during his visit to the clinic but cannot determine the discharge, accumulation or movement of the pills to another container. The measurement of prescriptions or prescriptions renewed in right time provides us information for adherence but no information is given whether the patient actually takes the medication or not. The direct observation may seem the most precise method; however, patients can hide their drugs in their mouths and reject them when they are not visible. The method of direct observation is most useful in measuring adherence through the presence to the appointment with the therapist. The electronic devices are accurate and a degree of patient cooperation is required. Blood and urine tests are expensive and impractical for use in the routine. Therefore, health professionals should be based on less direct and less sensitive methods. Interviews and questionnaires are flexible and practical methods to obtain information on the adherence to the treatment. However, the questionnaires may be difficult to use for patients with low levels of literacy. The Hill-Bone Compliance Scale (Hill-Bone) consists of three subscales (medication taking behavior, attendance in appointments and sodium intake) but it has limited generalization as it aims only to patients taking antihypertensive agents. Due to its high reliability (Cronbach's a 0.84 ) in populations of the black race, its use is recommended for studies in these populations (Kim,
Hill, Bone, \& Levine 2000; Krousel-Wood et al., 2013). The EightItem Morisky Medication Adherence Scale (MMAS-8) focuses on medication behavior and particularly in reduced use and negligence of taking medication (Tan, Patel, \& Chang 2014). The Medication Adherence Report Scale (MARS) focuses on beliefs and compliance barriers (Nguyen, Caze, \& Cottrell, 2014). It is based on the scale Drug Attitude Inventory (DAI) and explores the medication behavior and attitudes towards medication of patients with psychosis with high validity and reliability (Cronbach's a 0.75) (Thompson, Kulkarni, \& Sergejew, 2000).

In conclusion, each method, either objective or subjective, has strengths and limitations while some methods are more useful for some types of therapeutic recommendations than others (Achieng et al., 2013; Jimmy \& Jose, 2011).

In order to measure HD patient adherence, self-report instruments such as reliable and validates questionnaires are often used. Literature review revealed a small number of scales which can be used especially for hemodialysis patients. The Renal Adherence Attitudes Questionnaire (RAAQ) is a scale consisted of 26 items measuring attitudes toward adherence while the Renal Adherence Behaviour Questionnaire (RABQ) is a scale consisted of 25 items evaluating self-reported dietary and fluid adherence (Rushe \& McGee, 1998). The Dialysis Diet and Fluid Non-Adherence Questionnaire (DDFQ) (Vlaminck, Maes, Jacobs, Reyntjens, \& Evers, 2001) is a 4-item scale that assesses the level of adherence to fluid and dietary restriction for the past 14 days. However, the above tools do not explore the levels of attendance in HD session and medication use (Vlaminck et al., 2001). The End Stage Renal Disease Adherence Questionnaire (ESRD-AQ) is a 46 item questionnaire that addresses all aspects of adherence in HD. Nevertheless, the large number of questions should be considered (Kim, Evangelista, Phillips, Pavlish, \& Kopple, 2010). Finally, the Fluid Control in Hemodialysis Patients Scale (FCHPS) is a tool conducted on 2010 by Albayrak Cosar \& Cinar Pakyuz to focus on knowledge, behaviors, and attitudes of HD patients about the aspect of fluid restriction (Albayrak Cosar \& Cinar Pakyuz, 2010).

As a result, there is need of a reliable questionnaire to be addressed exclusively to patients undergoing HD measuring all aspects of adherence to HD regimen.

The aim of this study was to modify the Greek version of Simplified Medication Adherence Questionnaire (GR-SMAQ) for patients undergoing hemodialysis and explore its validity and reliability.

\section{Materials and Methods}

\section{Study design}

This study was a methodological study.

\section{Data collection}

The GR-Simplified Medication Adherence Questionnaire is a self-administered questionnaire that consists of 6 items of which four are dichotomous (Yes/No), one is Likert-type while one is open: (1) "Do you always take your medication at the appropriate time?" (2) "When you feel bad, have you ever discontinued taking your medication?" (3) "Have you ever forgotten to take your medication?" (4) "Have you ever forgotten to take your medications during the weekend?" (5) "In the last week, how many times did you fail to take your prescribed dose?" (6) "Since your last visit, how many whole days have gone by in that you did not take your 
medication?" (Theofilou, 2012).

In order to modify the GR-SMAQ for patients undergoing HD, a group of experts (5 patients undergoing HD, 3 hemodialysis nurses and 3 nephrologists) was asked about the degree of understanding, appropriateness and relevance of the scale. The following changes were made by the group: item 4 was amended as follows: "Have you ever forgotten to take your medications during the time interval between two dialysis sessions?" as patients undergoing HD may visit dialysis clinics on Saturday or on Sunday, also. Item 1 was removed as the right time of taking medication is likely to coincide with HD sessions. Finally, item 6 was, also, not included because patients undergoing HD visit dialysis clinics day by day. Four additional items were introduced to achieve the modification of the GR-SMAQ for patients undergoing HD. The above modifications were made with the consensus of Theofilou P. by whom the GR-SMAQ was translated into Greek language (Theofilou, 2012) for patients with lung cancer. Score range from $0-8$. Higher scores indicate greater adherence to HD regimen. It takes only 4 minutes to complete. The SMAQ was, firstly, constructed by Knobel et al. (2010) to measure adherence levels among HIV patients. It has, also, been used for evaluating adherence to phosphate binders among patients undergoing HD (Arenas, et al., 2010a; 2010b) and patients with early breast cancer (Oberguggenberger et al., 2012) respectively.

Finally, patients were given questionnaire related to demographic data (gender, age, educational level, job, marital status).

\section{Participants}

All 139 patients undergoing hemodialysis from three Dialysis Units of Athens and Peloponnese region, Greece, were asked to participate in this study. The inclusion criteria were: (a) age $>18$ years, (b) diagnosis of chronic kidney disease undergoing hemodialysis for at least 6 months (c) ability to write and read fluently the Greek language. The exclusion criteria were: history of mental illness and serious eye problems. Ultimately, a convenience sample of 107 patients met the mentioned criteria (response rate $76.9 \%$ ). The study was carried out during the period June 2016 November 2016.

\section{Ethical considerations}

The study was approved by the scientific councils of Dialysis Units (25/11/2015 and 24/10/13) and the Hellenic Data Protection Authority (Approval Number 1618, 11/02/2016). Patients, who met the criteria, after being informed about the aim of the study, gave their written consent and completed the above-mentioned questionnaires. Participants' anonymity was protected and safety of the material was maintained.

\section{Data analysis}

Content Validity Index (CVI) was used to assess the relevance of each question using a 4-point scale: $4=$ highly relevant, $3=$ relevant with minor adjustments, $2=$ minimally relevant and $1=$ not relevant. For the construct validity of the questionnaire the technique of factor analysis with the rotation method of axes was applied (Equamax by normalizing by Kaiser). Repeatability was evaluated by the method McNemar-Bowker. Internal consistency of the questionnaire was calculated through Cronbach's a coefficient while the significance level was set up at $5 \%$. Quantitative variables are described as mean ( \pm Standard Deviation) and qualitative variables as absolute frequencies and relative frequencies. The statistical analysis was performed using IBM SPSS Statistics version 20 (SPSS Inc., 2003, Chicago, USA).

\section{Results}

\section{Participants' characteristics}

In current study, 107 patients undergoing hemodialysis took part. Of these, $69 \%$ were males and $31 \%$ females. The mean age was $62( \pm 13)$ years old (Table 1). The frequency distribution of participants' responses to the GR-SMAQ-HD items is presented in Table 2 .

\section{Construct validity of the GR-SMAQ-HD}

CVI was 0.40 for item 1 ("Do you always take your medication at the appropriate time?") and item 6 ("Since your last visit, how many whole days have gone by in that you did not take your medication?"). Therefore, these items were removed. For the additional items (5-8) and the amended item (4) CVI was 0.90. Factor analysis of GR-SMAQ-HD items showed that all questions had a satisfactory correlation $(r>0.4)$ with at least one of the remaining questions. Also, all items were satisfactory sampling adequacy measures $(>0.6-$ Measures of Sampling Adequacy) and communalities ( $>0.5)$. For the evaluation of partial correlation coefficient the value of the KaiserMeyer-Olkin statistical criterion was 0.708 that is, also, considered satisfactory. Of the 8 questions, three factors emerged with eigenvalue greater than unity: "Medication adherence" (items 1, 2, 3, and 4), "Attendance at the HD session" (items 5 and 6) and "Diet/Fluid restrictions" (items 7 and 8). These three factors explain cumulatively the $63 \%$ of the total dispersion (Table 3 ).

\section{Reliability of GR-SMAQ-HD}

\section{Test-retest reliability}

The test-retest method was implemented for the reliability of the questionnaire. From the total of 107 patients, 17 of them com-

Table 1. Participants' characteristics $(\mathrm{N}=107)$.

\begin{tabular}{lc} 
Characteristic & N. $(\%)$ \\
Age (years), mean $( \pm$ SD) & $62( \pm 13)$ \\
Gender & \\
Males & $72(69)$ \\
Females & $33(31)$ \\
\hline Marital status & \\
Unmarried & $24(23)$ \\
Married & $6(6)$ \\
Divorced & $74(71)$ \\
Widowed & - \\
Number of children & \\
None & $27(26)$ \\
1 & $34(32)$ \\
2 & $15(14)$ \\
$\geq 3$ & $30(28)$ \\
\hline Educational level & \\
Primary school & $42(40)$ \\
Secondary school & $29(27)$ \\
High school & $18(17)$ \\
Undergraduate student & $3(3)$ \\
University & $14(13)$ \\
Job & \\
Unemployed & $17(16)$ \\
Employed & $29(27)$ \\
Retired & $60(57)$ \\
\hline
\end{tabular}

SD, standard deviation. 
pleted the GR-SMAQ-HD two weeks after the first administration. The repeatability evaluation was performed by controlling the McNemar-Bowker. No statistically significant difference was shown between the responses of the first and second reference to any question $(\mathrm{P} \geq 0.005)$ (Table 4$)$.

\section{Internal consistency}

Internal consistency was tested through Cronbach's alpha coefficient. The factor "Attendance at HD session" seems to have the highest Cronbach's alpha (0.856) while the factor "Medication adherence" follows with Cronbach's alpha 0.750 and "Diet/fluid restrictions" with Cronbach's alpha 0.717 . The internal consistency of the overall GR-SMAQ-HD scale consisting of 8 questions was: Cronbach's alpha $=0.742$. The whole scale and the subscales had Cronbach's alpha up to 0.71 that means that GR-SMAQ-HD has satisfactory internal consistency. Furthermore, the analysis showed that no substantial increase in Cronbach's alpha yet happened even if an item is deleted from the subscales. In conclusion, we could argue that all items had significant internal consistency each other.

The basic descriptive measures of location and dispersion and internal consistency of three factors (dimensions) of GR-SMAQHD are presented in Table 5. The mean GR-SMAQ-HD score was $6.29( \pm 1.82)$.

\section{Discussion}

This study was carried out in Athens and Peloponnese region and aimed to modify the GR-SMAQ scale for hemodialysis patients. The GR-SMAQ-HD is a modified form of GR-SMAQ and contains items to cover all aspects of adherence in hemodialysis. The initial form of SMAQ shows sufficient internal consistency among Spanish patients undergoing HD (Cronbach's alpha 0.70) (Arenas et al., 2010) and HIV patients (Cronbach's alpha=0.75) (Knobel et al., 2002).

\section{Measures of patient adherence}

Several measures have been constructed for the evaluation of patient adherence. Most of them focus on measure of medication and behavioral barriers to adherence (Lam \& Fresco, 2015). The Hill-Bone Compliance Scale has limited generalization as it aims only to patients taking antihypertensive agents (Kim et al., 2010). The Brief Medication Questionnaire can detect different types of non-adherence to the treatment (recurring or sporadic) (Svarstad, Chewning, Sleath, \& Claesson, 1999). The Medication Adherence Questionnaire is, also, known as the 4-Morisky Medication Adherence Scale (MMAS) and Morisky Scale is the most widely used scale. It has been used in a wide range of diseases and patients with low educational level (Culig \& Leppee, 2014). The Medication Adherence Report Scale (MARS) focuses on beliefs of adherence (Thompson, Kulkarni, \& Sergejew, 2000).

Regarding the questionnaires related to adherence among hemodialysis patients, the literature research refers to reliable scales: i) the Dialysis Diet and Fluid Non-Adherence Questionnaire (DDFQ) (Vlaminck et al., 2001) assess the frequency and degree of adherence to fluid and dietary restriction ii) the Renal Adherence Attitudes Questionnaire (RAAQ) measuring attitudes toward adherence and iii) the Renal Adherence Behavior Questionnaire (RABQ) measuring self-reported dietary (diet and fluid) adherence (Rushe \& McGee, 1998) and, finally, the End Stage Renal Disease Adherence Questionnaire (ESRD-AQ) (46

Table 2. The GR-SMAQ-HD scale ( $\mathrm{N}=107)$.

\section{GR-SMAQ-HID Items}

If you feel worse, do you stop taking yours medicines?

Yes

No

Do you ever forget to take you medicines?

Yes

No

Have you ever forgotten to take your medications during the time interval between two dialysis sessions?
Yes
No
$24(22)$
$83(78)$

How often did you not take your medicine over the last week?

$\begin{array}{lc}6-10 \text { times } & 2(2) \\ 3-5 \text { times } & 2(2) \\ 1-2 \text { times } & 28(26) \\ \text { Never } & 75(70)\end{array}$

Last month, how often did you shorten the session on your own initiative?

$\begin{array}{lc}4-5 \text { times } & 1(1) \\ 3 \text { times } & 2(2) \\ 2 \text { times } & 7(6) \\ 1 \text { time } & 18(17) \\ \text { I did not shorten the session } & 79(74)\end{array}$

Last month, on average how many minutes did you shorten the session with your own initiative?

$\begin{array}{lc}>30 & 1(1) \\ 21-30 & 8(7.5) \\ 11-20 & 7(6.5) \\ \leq 10 & 13(12) \\ \text { I did not shorten the session } & 78(73)\end{array}$

During the last week, how often did you follow the instructions on fluid restrictions?

Never $2(2)$

Rarely $12(11)$

Half of the time $15(14)$

Most of the time $38(36)$

Every time 40 (37)

During the last week, how often did you follow the instructions on diet?

$\begin{array}{lc}\text { Never } & 4(4) \\ \text { Rarely } & 7(6) \\ \text { Half of the time } & 14(13) \\ \text { Most of the time } & 45(42) \\ \text { Every time } & 37(35)\end{array}$

items) that explores all the aspects of adherence on HD, patients

Table 3. Loadings of the variables on the Factor Analysis of GRSMAQ-HD scale.

\begin{tabular}{lccc} 
Medication & $\begin{array}{c}\text { Factors } \\
\text { Attendance at }\end{array}$ & $\begin{array}{c}\text { Diet/Fluid } \\
\text { restrictions }\end{array}$ \\
Item 1 & 0.621 & -0.203 & 0.398 \\
Item 2 & 0.775 & 0.285 & 0.022 \\
\hline Item 3 & 0.860 & -0.092 & 0.131 \\
Item 4 & 0.692 & 0.348 & 0.167 \\
\hline Item 5 & 0.057 & 0.901 & 0.178 \\
Item 6 & 0.078 & 0.912 & 0.113 \\
\hline Item 7 & 0.157 & 0.107 & 0.871 \\
Item 8 & 0.085 & 0.213 & 0.821 \\
\hline
\end{tabular}

Method of extracting factors: Method of Principal Component; Rotation method of axes: Equamax by Kaiser normalization. 
beliefs and factors of non-adherence (Kim et al., 2010). None of these scales was designed exclusively for the study of adherence behavior in hemodialysis. On the contrary, the above scales focus more on identifying other aspects of adherence such as patients' attitudes, factors of nona-dherence or knowledge on hemodialysis regimen, (Kim et al., 2010; Rushe \& McGee, 1998).

In conclusion, several scales have been designed to assess adherence and, especially, its factors. However, we cannot support that there is an easy and short tool for measuring all views of adherence among patients undergoing HD (Smyth, Hartig, Hayes, \& Manickam, 2015).

In our study, we conducted factor analysis to identify possible items correlations. The factor analysis revealed three subgroups in the GR-SMAQ-HD. The first subgroup consisted of four items (items 1, 2, 3 and 4) that measure medication adherence and, already, exist in the first form of GR-SMAQ. The two items of the second group cover the aspect of attendance at HD session (items 5 and 6) while the last two items of the third group focus on Fluid/Diet restrictions (items 7 and 8). The three factors (Attendance to HD session, Diet/Fluid restrictions) revealed from this study are similar to those of the study of Kim and Evangelista (2013) using the Spanish version (End Stage Renal Disease Adherence Questionnaire, ESRD-AQ) and Portuguese (PESRDAQ) version of the End Stage Renal Disease Adherence Questionnaire (Poveda et al., 2016) while one factor (Diet/Fluid restrictions) is similar to the studies of Vlaminck et al. (2001) and Albayrak Cosar \& Cinar Pakyuz (2016).

The removal of items 1 and 6 of the initial GR-SMAQ allowed the factorial structure become simpler and more powerful. In particular, a group of experts (10 patients undergoing HD, 5 hemodialysis nurses and 3 nephrologists) suggested that item 1 ("Do you always take your medication at the appropriate time?") should be removed because the words "at the appropriate time" confuse patients because the correct hour of taking medication may coincides in time with the HD session. As far as item 6 is concerned ("Since your last visit, how many whole days have gone by in that you did not take your medication?"), the group of experts referred that it is not relevant to the specific group of dialysis patients. These changes reduced the obstacles for patients undergoing HD to complete the questionnaire and made it easier to interpret because all items can be considered reliable and study adherence.

Factor analysis confirms that the GR-SMAQ-HD fits the data well and explains $63 \%$ of the variance in all dimensions of adherence to HD regimen that is good for a brief, self-administered questionnaire.

The test-retest method was performed in order to check the repeatability. According to the literature (Streiner, Norman, \& Cairney, 2003), two to fourteen days are required between the first and second time of administration. Patients found the questionnaire easy to complete, understandable and none required any explanation of questions.

\section{Implication for practice}

The main benefit of using GR-SMAQ-HD is to identify the levels of adherence in order to target for intervention. Moreover, a clinician could identify especially the dimension in that a patient on hemodialysis is not adherent. The need for early identification and assessment of non-adherence is vital for patients undergoing hemodialysis (Ghimire, Castelino, Lioufas, Peterson, \& Zaidi, 2016; Karamanidou et al., 2008). However, given the complexity of adherence's pathogenesis and the lack of multidimensional tools, the effective intervention by HD nurses is difficult.
Therefore, nurses and health care professionals of hemodialysis units and renal clinics should be suspicious in the detection of nonadherence in order to improve the health care plan and help patients to develop coping strategies (Davison \& Cooke, 2015). Due to their contact with patients undergoing HD, hemodialysis nurses could improve adherence levels building a trusting relationship with patients through patient-center approach. It is well known that interventions should focus on patient education and counseling and that the higher the knowledge level the highest the adherence and quality of life levels (Barnes, Hancock, \& Dainton, 2013; Cowperthwaite, Schutt-Aine, Herranen, \& Sorribes, 2012; Sandlin, Bennett, Ockerby, \& Corradini, 2013).The GR-SMAQHD can be used in future research as a short, multidimensional adherence scale to successfully recognize the most useful areas for intervention aiming at improving patient outcomes. In addition, it could be beneficial if GR-SMAQ-HD compared with other more objective measures in order to investigate sensitivity and specificity more.

\section{Strengths and limitations}

GR-SMAQ-HD is a short tool that can be completed within minutes, while it is easily assessed, thus allowing health professionals to measure and evaluate adherence easily. Also, it is the first scale for Greek patients undergoing HD measuring adherence levels. As already mentioned, the study was conducted in Dialysis Units of Athens and Peloponnese region. Therefore, results cannot be generalized for patient undergoing HD. Moreover, the questionnaires were administered during HD session so the presence of physicians, nurses and others may have influenced the responses of participants.

Table 4. Test-retest reliability of GR-SMAQ-HD (two week period between test-retest, $\mathrm{n}=17$ ).

\begin{tabular}{lccc}
$\begin{array}{l}\text { GR-SMAQ-HD } \\
\text { Items }\end{array}$ & $\begin{array}{c}\text { Test }(\mathrm{A}), \\
\text { mean }( \pm \mathrm{SD})\end{array}$ & $\begin{array}{c}\text { Re-test }(\mathrm{B}), \\
\text { mean }( \pm \mathrm{SD})\end{array}$ & P-value \\
Item 1 & $0.88( \pm 0.5)$ & $0.89( \pm 0.41)$ & 1.000 \\
Item 2 & $0.87( \pm 0.3)$ & $0.87( \pm 0.33$ & 1.000 \\
\hline Item 3 & $0.85( \pm 0.24)$ & $0.88( \pm 0.22)$ & 1.000 \\
Item 4 & $0.90( \pm 0.21)$ & $0.89( \pm 0.19)$ & 1.000 \\
\hline Item 5 & $0.98( \pm 0.10)$ & $0.97( \pm 0.11)$ & 1.000 \\
Item 6 & $0.97( \pm 0.11)$ & $0.97( \pm 0.10)$ & 1.000 \\
\hline Item 7 & $0.89( \pm 0.12)$ & $0.88( \pm 0.13)$ & 0.368 \\
Item 8 & $0.88( \pm 0.11)$ & $0.87( \pm 0.10)$ & 0.317
\end{tabular}

Table 5. Descriptive characteristics and internal consistency of the GR-SMAQ-HD ( $\mathrm{N}=107)$.

\begin{tabular}{lccccc} 
& Min. Max. & Mean & SD & Cronbach's a \\
Total score & 1 & 8 & 6.29 & 1.82 & 0.742 \\
Medication & 0 & 4 & 3.04 & 1.15 & 0.750 \\
\hline Attendance at HD session & 0 & 2 & 1.76 & 0.58 & 0.856 \\
Diet/Fluid restriction & 0 & 2 & 1.50 & 0.74 & 0.717 \\
\hline
\end{tabular}

SD, Standard Deviation. 


\section{Conclusions}

Through the study of the psychometric properties of the GRSMAQ-HD, its validity was found to be highly satisfactory. This questionnaire seems to be an easy and appropriate tool to conduct studies exploring adherence to HD regimen. Therefore, it would be useful for widespread use in future studies to validate this questionnaire with a larger sample of patients undergoing HD.

\section{References}

Achieng, L., Musangi, H., Billingsley, K., Onguit, S., Ombegoh, E., Bryant, L., ... Keiser, P. (2013). The use of pill counts as a facilitator of adherence with antiretroviral therapy in resource limited settings. PLoS One, 8(12), e67259. doi: 10.1371/journal.pone.0067259

Albayrak Cosar, A., \& Cinar Pakyuz, S. (2016). Scale development study: the fluid control in hemodialysis patients. Japanese Journal of Nursing Science, 13, 174-182.

Arenas, M.D., Malek, T., Álvarez-Ude, F., Gil, M.T., Moledous, A. \& Reig-Ferrer, A. (2010a). Phosphorus binders: preferences of patients on haemodialysis and its impact on treatment compliance and phosphorus control. Nefrologia, 30, 522-530.

Arenas, M.D., Malek, T., Gil, M.T., Moledous, A., Alvarez-Ude, F. \& Reig-Ferrer, A., (2010b). Challenge of phosphorus control in hemodialysis patients: a problem of adherence? Journal of Nephrology, 23, 525-534.

Barnes, T., Hancock, K. \& Dainton, M. (2013).Training nurses to support greater patient engagement in haemodialysis. Journal of Renal Care, 39 Suppl 2:10-8.

Bellizzi V., Cupisti, A., Locatelli, F., Bolasco, P., Brunori, G., Cancarini, G., ... Conservative Treatment of CKD study group of the Italian Society of Nephrology (2016). Low-protein diets for chronic kidney disease patients: the Italian experience. BMC Nephrology, 17, 77.

Block, G.A., Klassen, P.S., Lazarus, J.M. Ofsthun, N., Lowrie, E.G. \& Chertow, G.M. (2004). Mineral metabolism, mortality, and morbidity in maintenance hemodialysis. Journal of the American Society of Nephrology, 15, 2208-2218.

Cowperthwaite, J., Schutt-Aine, R., Herranen, M. \& Sorribes, M.P. (2012). Introduction of a competency based haemodialysis education programme: 5 years experience. Journal of Renal Care, 38, 162-169.

Culig, J. \& Leppée, M. (2014). From Morisky to Hill bone; selfreports scales for measuring adherence to medication. Collegium Antropologicum, 38, 55-62.

Davison, I. \& Cooke, S. (2015). How nurses' attitudes and actions can influence shared care. Journal of Renal Care, 41, 96-103.

Durose, C.L., Holdsworth, M., Watson, V. \& Przygrodzka, F. (2004). Knowledge of dietary restrictions and the medical consequences of noncompliance by patients on hemodialysis are not predictive of dietary compliance. Journal of the American Dietetic Association, 104, 35-41.

Erlingmark, J., Hedström, M. \& Lindberg, M. (2016). Nurse staffing and renal anaemia outcomes in haemodialysis care. Journal of Renal Care, 42, 185-189.

Estrella, M.M., Jaar, B.G., Cavanaugh, K.L., Fox, C.H., Perazella, M.A., Soman, S.S., ..., Choi, M.J. (2013). Perceptions and use of the national kidney foundation KDOQI guidelines: a survey of U.S. renal healthcare providers. BMC Nephrology, 14, 230. doi: 10.1186/1471-2369-14-230.
Ghimire, S., Castelino, R.L., Lioufas, N.M., Peterson, G.M. \& Zaidi, S.T. (2015). Nonadherence to medication therapy in haemodialysis patients: A Systematic Review. PLoS One, 10(12), e0144119. doi: 10.1371/journal.pone.0144119

Goodman, W.G. (2002). Vascular calcification in end-stage renal disease. Journal of Nephrology, 15 suppl 6, S82-S85.

Ibrahim, S., Hossam, M. \& Belal, D. (2015). Study of non-compliance among chronic hemodialysis patients and its impact on patients' outcomes. Saudi Journal of Kidney Diseases and Transplantation, 26, 243-249.

Ikonomou, M., Skapinakis, P., Balafa, O., Eleftheroudi, M., Damigos, D. \& Siamopoulos, K.C. (2015). The impact of socioeconomic factors on quality of life of patients with chronic kidney disease in Greece. Journal of Renal Care, 41, 239246.

Jimmy, B. \& Jose, J. (2011). Patient medication adherence: measures in daily practice. Oman Medical Journal, 26, 155-159.

Kammerer, J., Garry, G., Hartigan, M., Carter, B. \& Erlich, L. (2007). Adherence in patients on dialysis: strategies for success. Nephrology Nursing Journal, 34, 479-486.

Karamanidou, C., Clatworthy, J., Weinman, J. \& Horne, R. (2008). A systematic review of the prevalence and determinants of nonadherence to phosphate binding medication in patients with end-stage renal disease. BMC Nephrology, 9:2. doi: 10.1186/1471-2369-9-2.

Kim, M., Hill, M., Bone, L., \& Levine, D. (2000). Development and testing of the Hill-Bone Compliance to High Blood Pressure Therapy Scale. Progress In Cardiovascular Nursing, $15,90-96$.

Kim, Y. \& Evangelista, L.S. (2013). Development and cultural adaptation of the Spanish Version of the End Stage Renal Disease Adherence Questionnaire (SESRD-AQ). Nephrology Nursing Journal, 40, 493-507.

Kim, Y., Evangelista, L.S., Phillips, L.R., Pavlish, C. \& Kopple, J.D. (2010). End-Stage Renal Disease Adherence Questionnaire (ESRD-AQ): testing the psychometric properties in patients receiving in-center hemodialysis. Nephrology Nursing Journal, 37, 377-393.

Knobel, H., Alonso, J. Casado, J.L., Collazos, J., González, J., Ruiz, I., ..., GEEMA Study Group (2002). Validation of a simplified medication adherence questionnaire in a large cohort of HIV-infected patients: the GEEMA Study. AIDS, 16, 605-613.

Krousel-Wood, M., Joyce, C., Holt, E., Levitan, E., Dornelles, A., Webber, L., \& Muntner, P. (2013). Development and evaluation of a self-report tool to predict low pharmacy refill adherence in elderly patients with uncontrolled hypertension. Pharmacotherapy: The Journal of Human Pharmacology and Drug Therapy, 33, 798-811.

Kutner, N.G., Zhang, R., \& McClellan, W.M. (2002). Psychosocial predictors of non-compliance in haemodialysis and peritoneal dialysis patients. Nephrology Dialysis Transplantation, 17, 9399.

Lam, W.Y. \& Fresco, P. (2015). Medication adherence measures: an overview. BioMed Research International 217047. doi: $10.1155 / 2015 / 217047$.

Lee, S.W. (2012). Sodium balance in maintenance hemodialysis. Electrolytes \& Blood Pressure, 10, 1-6.

Leggat, J.E., Orzol, S.M., Hulbert-Shearon, T.E., Golper, T.A., Jones, C.A., Held, P.J. \& Port, F.K. (1998). Noncompliance in hemodialysis: predictors and survival analysis. American Journal of Kidney Diseases, 32, 139-145.

Lòpez-Gòmez, J.M., Villaverde, M., Jofre, R., Rodriguez-Benítez, P. \& Pérez-García R. (2005). Interdialytic weight gain as a 
marker of blood pressure, nutrition, and survival in hemodialysis patients. Kidney International, Suppl 93, S63-S68

Nguyen, T., Caze, A., \& Cottrell, N. (2014). What are validated self-report adherence scales really measuring? A systematic review. British Journal of Clinical Pharmacology, 77, 427445. http://dx.doi.org/10.1111/bcp.12194

Oberguggenberger, A.S., Sztankay, M., Beer, B., Schubert, B., Meraner, V., Oberacher H..., Hubalek, M. (2012). Adherence evaluation of endocrine treatment in breast cancer: methodological aspects. BMC Cancer, 12, 474. doi: 10.1186/1471-2407-12-474.

Poveda, V., Amado, L., Filgueiras, M., Teixeira, L., Miranda, V., Santos-Silva, A., ..., Costa, E. (2016) End-stage renal disease adherence questionnaire: translation and validation to the Portuguese language, Renal Failure, 38(10), 1633-1638.

Rushe, H. \& McGee, H.M. (1998). Assessing adherence to dietary recommendations for hemodialysis patients: the Renal Adherence Attitudes Questionnaire (RAAQ) and the Renal Adherence Behaviour Questionnaire (RABQ). Journal of Psychosomatic Research, 45, 149-157.

Sandlin, K., Bennett P.N., Ockerby, C. \& Corradini, AM. (2013). The impact of nurse-led education on haemodialysis patients' phosphate binder medication adherence. Journal of Renal Care, 39, 12-18.

Segall, L., Nistor, I. \& Covic, A. (2014). Heart failure in patients with chronic kidney disease: A systematic integrative review. BioMed Research International, 937398. http://doi.org/10.1155/2014/937398

Smyth, W., Hartig, V., Hayes, M. \& Manickam, V. (2015). Patients' adherence to aspects of haemodialysis regimens in tropical north Queensland, Australia. Journal of Renal Care, 41, 110-118.

Streiner, D.L. Norman, G.R. \& Cairney, J. (2014). Health measurement scales: A practical guide to their development and use $\left(5^{\text {th }}\right.$ ed.). New York: Oxford University Press Inc.

Svarstad, B.L., Chewning, B.A., Sleath, B.L. \& Claesson, C.
(1999). The Brief Medication Questionnaire: a tool for screening patient adherence and barriers to adherence. Patient Education and Counseling, 37, 113-24.

Szczech, L.A., Reddan, D.N., Klassen, P.S., Coladonato, J, Chua, B, Lowrie, E., ..., Owen, W.F.Jr. (2003). Interactions between dialysis-related volume exposures, nutritional surrogates and mortality among ESRD patients. Nephrology Dialysis Transplantation, 18, 1585-1591.

Tan, X., Patel, I., Chang, J. (2014). Review of the four item Morisky Medication Adherence Scale (MMAS-4) and eight item Morisky Medication Adherence Scale (MMAS8). University of Minnesota, College of Pharmacy. Retrieved from the University of Minnesota Digital Conservancy, http://hdl.handle.net/11299/171823.

Theofilou, P. (2012). Results from the translation and cultural adaptation of the Greek Simplified Medication Adherence Questionnaire (GR-SMAQ) in patients with lung cancer. Journal of Clinical Trials, 3, 2. http://dx.doi.org/10.4172/2167-0870.1000133

Thompson, K., Kulkarni, J. \& Sergejew, A.A. (2000). Reliability and validity of a new Medication Adherence Rating Scale (MARS) for the psychoses. Schizophrenia Research, 42, 241-247.

van der Meer, I.M., Ruggenenti, P. \& Remuzzi, G., (2010). The diabetic CKD patient - a major cardiovascular challenge. Journal of Renal Care, 36 Suppl 1:34-46.

Vlaminck, H., Maes, B., Jacobs, A., Reyntjens, S. \& Evers, G. (2001). The Dialysis Diet and Fluid Non-adherence Questionnaire: Validity testing of a self-report instrument for clinical practice. Journal of Clinical Nursing, 10, 707-715.

Zyga, S., Alikari, V., Sachlas, A., Fradelos, E.C., Stathoulis, J., Panoutsopoulos, G., ..., Lavdaniti, M. (2015). Assessment of fatigue in end stage renal disease patients undergoing hemodialysis: prevalence and associated factors. Medical Archives, 69, 376-380. 